Diesen Ergebnissen entsprechend ist in Rechnung gebracht:

$$
\text { Argelander - Schjellerup }=\text { +o.s. I +0.3 }
$$

Der kleine Unterschied in der Zahl der Sterne bei der Ordnung nach Rectascension und nach Declination rührt davon her, dass einige Zonen, in die nur ein Stern fiel, nicht berücksichtigt wurden.

Durch den Aufsatz von Herrn Oertel in den A. N. Nr. 2990 wurde ich nun darauf aufmerksam gemacht, dass bei unserer Vergleichung von Argelander und Schjellerup die in der Einleitung zu Bonner Beobachtungen Bd.VI S. IX Z. 26 gemachte Bemerkung übersehen worden ist, wonach die vor und nach dem Anfang des Jahres 1859 angestellten Beobachtungen Argelander's in Declination auf verschiedenen Grundlagen beruhen und sich um 0.4 von einander unterscheiden. Herr Oertel giebt ferner an, dass er die vor diesem Zeitpunkte angestellten Rectascensionen um den Unterschied des Catalogus Aboënsis und der Tabulae Reductionum von Wolfers, nämlich um 0.56 , verbessert habe; indess scheint mir dies nicht in Uebereinstimmung mit der auf Seite VI Zeile 22 von Argelander gemachten Angabe zu stehen, wonach für den hier in Betracht kommenden Theil des Catalogs, nämlich ftir die Sterne bis zu $-14^{\circ}$ Declination, die Verbesserung von 0.06 von ihm schon angebracht ist.

Um über diese Unterschiede noch weitere Aufschltusse zu erhalten, hat Dr. Buschbaum in letzter Zeit innerhalb der Grenzen von $-15^{\circ}$ und $+15^{\circ}$ Declination, um welche es sich bei den Klinkerfues'schen Zonen handelt, eine Vergleichung der vor und nach 1859 angestellten Sternörter des Catalogs in den Bonner Beob. Bd.VI ausgefuhrt und gefunden :

Beobachtungen nach I 859 - vor I 859 +o.or 5 -o".39.

Diese Untersuchung ist nur zur Beantwortung der Frage angestellt, ob die erwähnten Unterschiede zwischen den Beobachtungen beider Zeiträume stattfinden und nicht etwa zur Ermittelung genauer Reductionsgrössen, es ist deshalb auch hier keine Rücksicht auf die Vollständigkeit oder Unvollständigkeit der Beobachtungen genommen und wie oben wieder 0.49 und 4.9 als Grenze der verwendbaren Unterschiede angesetzt. Wenn auch die Endwerthe noch mit nicht unbeträchtlichen mittleren Fehlern behaftet sind, so geht doch aus dieser Rechnung mit einiger Sicherheit hervor, dass die erwähnte Reduction von 0.06 in Rectascension nicht mehr anzubringen ist und dass sich die Declinationen wirklich um etwa $0^{\prime \prime} 4$ unterscheiden.

In Anbetracht der bei den Klinkerfues'schen Zonenbeobachtungen erreichten Genauigkeit und des Umstandes, dass die Rechnungen bis zum Herstellen des Manuscripts für den Druck bereits vollständig abgeschlossen waren, haben wir uns nicht veranlasst gesehen, noch nachträglich den nach Argelander's Ansicht nicht einmal ganz sicheren Unterschied von $0.4 \mathrm{zu}$ berücksichtigen und deshalb die vielen mühsamen Ausgleichungen zur Verwandlung der Scalenablesungen in Declination (vergl. darüber meinen Bericht in der Vierteljahrsschrift der Astr. Ges.) zu verbessern, sondern wir müssen uns mit dem Hinweis begnügen, dass bei der Aufstellung unseres ,Fundamental-Catalogse nach Argelander, Schjellerup und Yarnall nach einer Abzählung nahezu gleich viele Argelander'sche Beobachtungen vor und nach 1859 verwandt sind und daher, wenn der Catalog als auf Wolfers beruhend bezeichnet wird, die Abweichungen davon in Declination im Durchschnitt nicht grösser als die Hälfte des erwähnten Unterschiedes, also etwa o". 2 betragen können.

Wegen Vernachlässigung der Verbesserung von 0.4 kann unsere Vergleichung keine Prufung der von Herrn Oertel aufgefundenen auffallenden Abhängigkeit der Declinations - Differenzen BB.VI - Sj. abgeben und da unsere Rechnungen dieselben nicht so stark ausgesprochen zeigen, so haben wir uns damals mit der Annahme eines einfachen Mittelwerthes begnügt. Als Mittelwerth für die Unterschiede in Rectascension findet Herr Oertel +o.147, dagegen geben unsere Rechnungen +o:ri4 ; der Unterschied von 0.033 deutet wieder darauf hin, dass die Verbesserung von 0.06 für einen Theil der Sterne angebracht und wie bemerkt, die Zahl der Sterne ziemlich gleichmässig auf den Zeitraum vor und nach 1859 vertheilt ist.

Göttingen 1890 December.

Wilhelm Schur.

\title{
Ueber die Münchener Sterne der Bonner Südlichen Durchmusterung.
}

\section{Von Dr. F. Bauschinger.}

Bekanntlich hat Herr Geheimrath Schönfeld seine südliche Durchmusterung mit den Lamont'schen Sterncatalogen verglichen und jene Sterne der SD., für welche nur in diesen Catalogen genauere Meridianbestimmungen vorlagen, mit $M$ bezeichnet. Durch das kürzlich herausgegebene - Erste Münchener Sternverzeichniss (Neue Annalen der Münchener Sternwarte Band I) sind nun aber diese Cataloge antiquirt, da dasselbe die sämmtlichen brauchbaren Positi. onen dieser letzteren in verbesserter Reduction wiedergiebt. Es ist daher angezeigt, die Angaben der SD. mit dieser neuen Ausgabe der älteren Münchener Zonenbeobachtungen in Einklang zu bringen; zu dem Ende sind einige Verbesserungen in den Angaben der SD., soweit sie $M$ betreffen, nothwendig, und ich erlaube mir, dieselben im Folgenden mitzutheilen mit dem Bemerken, dass hier nicht etwa Fehler dieses ausgezeichneten Werkes vorliegen, sondern lediglich Aenderungen, welche durch die Neuredaction der Lamont'schen Verzeichnisse bedingt sind.

1. In folgenden Nummern der SD. ist $M$ zu streichen, wenn man diesen Nachweis auf die Neuausgabe der Lamont'schen Zonen $\left(M_{1}\right)$ bezieht; es findet sich fur diese Nummern aber in dem demnächst erscheinenden $\gg$ Zweiten 
Münchener Sternverzeichnisse $\left(\mathrm{M}_{2}\right)$ eine neue Meridianbeobachtung :

$$
\begin{array}{ll}
-2.276 & -8.4973 \\
-6.1772 & -11.3232 \\
-6.3840 & -12.5492 \\
-6.5305 & -13.3073 \\
-7.4882 & -14.6523
\end{array}
$$

2. In folgenden Nummern ist $M$ zu streichen, da der betreffende Stern in $\mathbf{M}_{1}$ nicht mehr vorkommt; auch $M_{2}$ enthält keine Beobachtungen für diese Sterne :

$$
\begin{array}{ll}
-2.3670 & -6.3239 \\
-2.4948 & -6.4368 \\
-3.3054 & -14.3748 \\
-3.3113 & -\mathrm{r} 8.5732 \text { (muss A heissen) } \\
-6.3216 &
\end{array}
$$

\begin{tabular}{|c|c|c|c|c|c|c|c|c|c|c|}
\hline Datum & M.Z.Dresd. & $\Delta \alpha$ & $\Delta \delta$ & Vgl. & $\alpha$ app. & $\log p \cdot \Delta$ & $\delta$ app. & $\log p .4$ & Red. ad l. app. & * \\
\hline & & & & & & & & & & \\
\hline $\begin{array}{l}\text { Sept. } 23 \\
\text { Oct. } 4\end{array}$ & 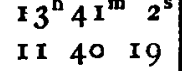 & - & $\begin{array}{rr}-8 & 0.0 \\
-14 & 25 \cdot 3\end{array}$ & $\begin{array}{c}12.4 \\
7\end{array}$ & - & $\stackrel{9.291}{-}$ & $\begin{array}{r}-21318.0 \\
-30 \\
-30.7\end{array}$ & $\begin{array}{l}0.847 \\
0.853\end{array}$ & $\begin{array}{l}+2.43+15.8 \\
+2.45+15.8\end{array}$ & 2 \\
\hline $\begin{array}{c}\text { Nov. I } 7 \\
1890\end{array}$ & $\begin{array}{llll}8 & 50 & 23\end{array}$ & +114.84 & - $54^{2.2}$ & I5.5 & $2324 \quad 56.39$ & $9.07 \mathrm{I}$ & $-4 \times 753.7$ & 0.859 & $+2.10+13.5$ & 3 \\
\hline Nov. 18 & $\begin{array}{lll}11 & 28 & 21\end{array}$ & +341.16 & - I 50.7 & 15.5 & $44 \quad 29.24$ & $9.162_{n}$ & $+225^{6} \quad 57 \cdot 3$ & $\mathbf{I}$ & $+3.30+8.2$ & 4 \\
\hline Dec. I & $\begin{array}{lll}8 & 34 & 23\end{array}$ & to 42.17 & +855.3 & 27.9 & $\begin{array}{lll}4 & 33 & 17.03\end{array}$ & $9 \cdot 484 n$ & $+223^{8} 35.7$ & 0.692 & $+3.49+9.2$ & 5 \\
\hline 14 & $8 \quad 5 \circ \quad 34$ & +349.06 & -1341.2 & 18.6 & $42 \times 48.25$ & $9.294 n$ & +22 I5 41.8 & 0.653 & $+3.5^{8}+10.7$ & 6 \\
\hline 15 & 91816 & +258.47 & -1531.8 & 18.6 & $420 \quad 57.66$ & $9.162 n$ & +221351.2 & 0.640 & $+3.59+10.7$ & 6 \\
\hline
\end{tabular}

\begin{tabular}{|c|c|c|c|}
\hline * & a 1889.0 & $\delta \times 889.0$ & Autorität \\
\hline $\mathbf{I}$ & $23^{\mathrm{h}} 43^{\mathrm{m}} \quad 2^{\mathrm{s}} .03$ & $-2^{\circ} 5^{\circ} 33^{\prime \prime} 8$ & Berl. Mer. (Rü \\
\hline 2 & 234 I 47.42 & $-\begin{array}{lll}-2 & 46 & 1.2\end{array}$ & Berl. Mer. $\left(W_{1}\right)$ \\
\hline 3 & $2323 \quad 39.45$ & $-4 \begin{array}{lll}-4 & 125.0\end{array}$ & $\begin{array}{l}\text { 1/2 (Pulk.A.N. } 1477+\text { Berl. } \\
\text { A.N. 1637) }\end{array}$ \\
\hline
\end{tabular}

München 1890 December.

\begin{tabular}{|c|c|c|c|}
\hline * & $\alpha 1890.0$ & $\delta 1890.0$ & Autorität \\
\hline 4 & $4^{\mathrm{h}} 40^{\mathrm{m}} 44^{\mathrm{s}} \cdot 78$ & $+22^{\circ} 5^{\prime} 39: 8^{\prime}$ & AG. Berlin $\left(W_{2}\right)$ \\
\hline 5 & $\begin{array}{l}4231 \cdot 38 \\
47\end{array}$ & $+22293 \mathbf{I . 1}$ & $\begin{array}{l}\text { BB.V1+22:724 } \\
\text { AG. Berlin }(E D, 1880.6)\end{array}$ \\
\hline & 4850.01 & $1+22 \quad 2912.3$ & 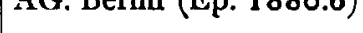 \\
\hline
\end{tabular}

\section{Beobachtungen des Planeten (24) Themis}

am Fadenmikrometer des r inch. Aequatoreal der Privatsternwarte in Dresden von Dr. B. von Engelhardt.

1889 Sept. 23. Dunst. Unruhig. Planet 11.0 Grösse. - Oct. 4. Heller Mondschein. Planet schwach. Beob. achtung etwas unsicher. - Nov. 1 7. Luft ziemlich gut. Planet 12.5 Grösse.

1890 Nov. 18. Luft schlecht; zuletzt ganz trübe. - Dec. I und 15. Luft ziemlich gut. - Dec. 14. Luft gut.

Mittlęre Oerter der Vergleichsterne bezogen auf den jedesmaligen Jahresanfang.

Die beiden Berliner Meridianbeobachtungen der Sterne $\mathbf{I}$ und 2 verdanke ich der Freundlichkeit des Herrn Dr. Küstner; die AG. Positionen der Sterne 4 und 6 wurden mir von Herrn Prof. E. Becker gütigst mitgetheilt. Für

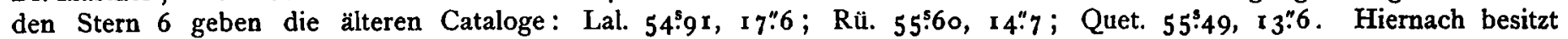
der Stern wahrscheinlich eine jährliche Eigenbewegung von -0.06 in Decl.; sollte sich diese durch spätere Beobachtungen bestätigen, so ist der angenommene Sternort um - o"6 in Decl. zu corrigiren.

Vergleichung der Beobachtungen mit den Elementen von Prof. Krueger im Sinne (B - R) :

\begin{tabular}{c|c|c}
\hline 1889 & $\Delta \alpha$ & $\Delta \delta$ \\
\hline Sept. 23 & -0.83 & +0.2 \\
Oct. 4 & - & +0.2 \\
Nov. 1 7 & -0.96 & -1.1
\end{tabular}

Dresden I 89 I Januar.

\begin{tabular}{r|c|c}
\hline \multicolumn{1}{c|}{1890} & $\Delta \alpha$ & $\Delta \delta$ \\
\hline Nov. 18 & -0.36 & -1.0 \\
Dec. 1 & -0.53 & -2.9 \\
14 & -0.33 & +0.2 \\
15 & -0.51 & -0.2
\end{tabular}

B. von Engelhardt. 